\title{
A dispersive backend design for the 'double-Fourier' interferometer BETTII
}

\section{Arnab Dhabal, Stephen A. Rinehart, John E. Mentzell, Maxime J. Rizzo, Lee Mundy, et al.}

Arnab Dhabal, Stephen A. Rinehart, John E. Mentzell, Maxime J. Rizzo, Lee Mundy, Dale Fixsen, Todd Veach, Roser Juanola-Parramon, Jordi Vila Hernandez de Lorenzo, "A dispersive backend design for the 'double-Fourier' interferometer BETTII," Proc. SPIE 10701, Optical and Infrared Interferometry and Imaging VI, 107011G (9 July 2018); doi: 10.1117/12.2312138

Event: SPIE Astronomical Telescopes + Instrumentation, 2018, Austin, Texas, United States 


\title{
A dispersive backend design for the 'double-Fourier' interferometer BETTII
}

\author{
Arnab Dhabal ${ }^{\mathrm{a}, \mathrm{b}}$, Stephen A. Rinehart ${ }^{\mathrm{a}}$, John E. Mentzell ${ }^{\mathrm{a}}$, Maxime J. Rizzo ${ }^{\mathrm{a}}$, Lee Mundy ${ }^{\mathrm{b}}$, \\ Dale Fixsen $^{a}$, Todd Veach ${ }^{a}$, Roser Juanola-Parramon ${ }^{a}$, and Jordi Vila Hernandez de Lorenzo ${ }^{a}$

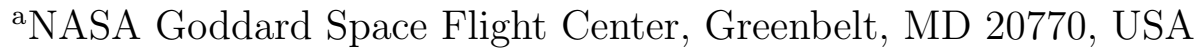 \\ ${ }^{\mathrm{b}}$ Department of Astronomy, University of Maryland, College Park, MD 20741, USA
}

\begin{abstract}
BETTII (Balloon Experimental Twin Telescope for Infra-red Interferometry) is designed to provide high angular resolution spectroscopic data in the far-infrared (FIR) wavelengths. The most significant limitation for BETTII is its sensitivity; obtaining spectral signal-to-noise ratio $>5$ in $<10$ minutes requires sources $>13 \mathrm{Jy}$. One possible way to improve the signal-to-noise ratio (SNR) for future BETTII flights is by reducing the spectral bandwidth post beam-combination. This involves using a dispersive element to spread out a polychromatic point source PSF on the detector array, such that each pixel corresponds to a small fraction of the bandwidth. This results in a broader envelope of the interferometric fringe pattern allowing more fringes to be detected, and thereby improving the spectral SNR. Here we present the analysis and optical design of the dispersive backend, discussing the tradeoffs and how it can be combined with the existing design.
\end{abstract}

Keywords: spatio-spectral interferometry, far infra-red, delay line, capacitive sensor, interferometric simulation, metrology, alignment

\section{INTRODUCTION}

The balloon-borne interferometer BETTII combines the techniques of Michelson Interferometry and Fourier Transform Spectroscopy (FTS) to obtain spatially resolved far-infrared (FIR) spectra of protostellar clusters. ${ }^{1}$ The design of BETTII involves collecting light from two apertures 8 meters apart, modulating the optical path lengths between the two arms systematically, combining the beams and focusing them onto detectors. BETTII has two bands in the FIR: 30-55 $\mu \mathrm{m}$ short wavelength (SW) band and 55-100 $\mu \mathrm{m}$ long wavelength (LW) band respectively, and uses two $9 \times 9$ detector arrays per channel to record the interferograms. ${ }^{2,3}$

BETTII had its Engineering Flight in June 2017 from Palestine, TX (Figure 1). The flight allowed us to validate the optical system, retention of its alignment through flight, the stability of the metering truss, the gondola pointing system, thermal performance, flight electronics, software, ground communication, realtime monitoring and commanding of subsystems. The first flight results of BETTII can be found in these proceedings. ${ }^{4,5}$ A science flight was planned to follow the Engineering flight to study dense protostellar fields like S140. However, an anomaly at the end of the first flight, resulted in the payload separating from the parachute leading to a complete loss of the payload. The team is currently rebuilding the payload (BETTII-2) drawing heavily from the same design (Figure 2), but at the same time upgrading some aspects of the design to improve the performance, facilitate easier testing and/or reduce costs and risks.

The BETTII optical design is also being upgraded to improve the overall sensitivity. In this paper, we discuss this new optical design. A dispersive backend has been included in the optics train. With the help of mathematical formalism and interferometric simulations of BETTII observations, we explain how the dispersive element will improve the sensitivity of the instrument. We discuss the grating design and the resulting output at the image plane. The dispersive backend is the only fundamental optics design change in BETTII-2 compared to the flown version. However, we have also incorporated some changes to redistribute the thermal loading and for ground testing of the FIR optics. In the final section, we discuss these design changes.

Author contact: Arnab Dhabal, arnab.dhabal@nasa.gov

Optical and Infrared Interferometry and Imaging VI, edited by Michelle J. Creech-Eakman,

Peter G. Tuthill, Antoine Mérand, Proc. of SPIE Vol. 10701,107011G · C 2018 SPIE

CCC code: $0277-786 \mathrm{X} / 18 / \$ 18 \cdot$ doi: $10.1117 / 12.2312138$ 


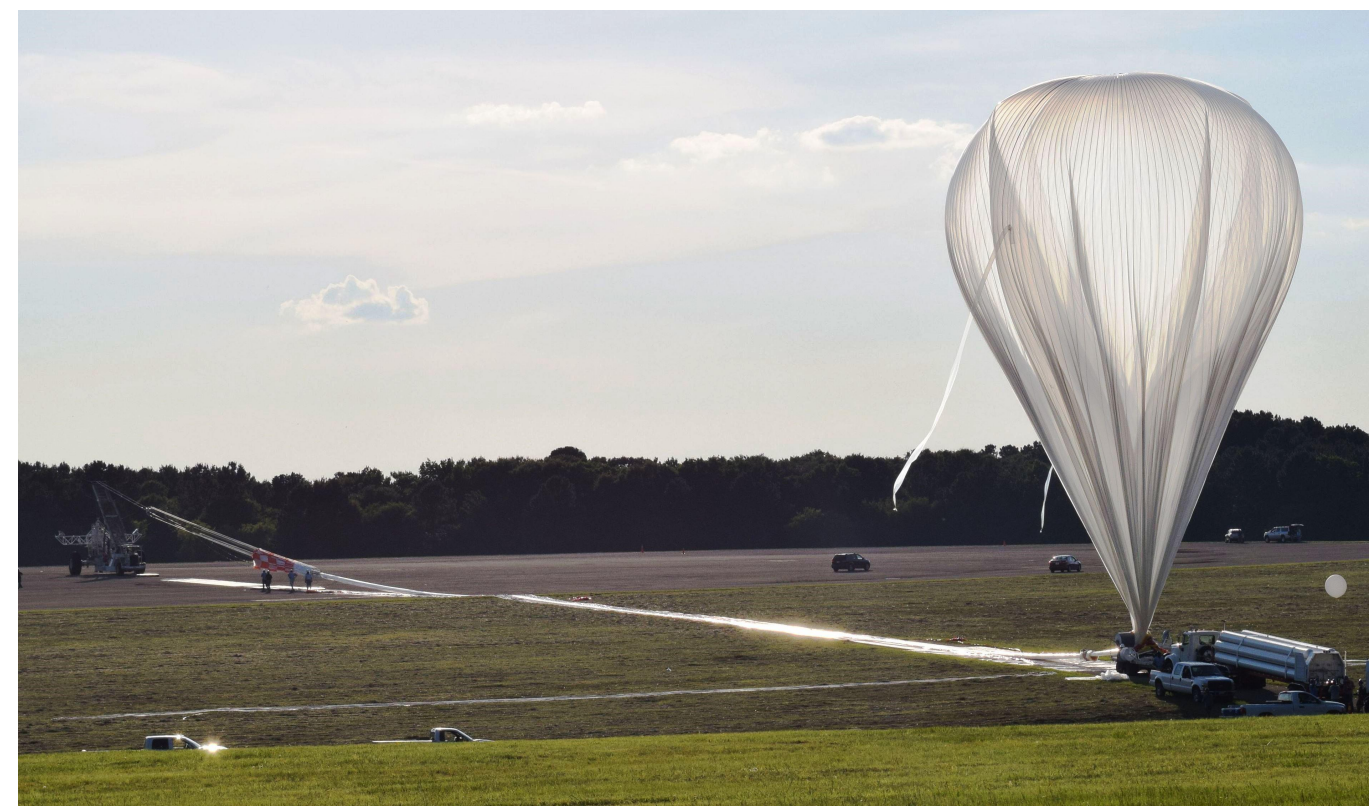

Figure 1. BETTII connected to the Helium balloon via the flight train, prior to launch from CSBF Palestine, TX

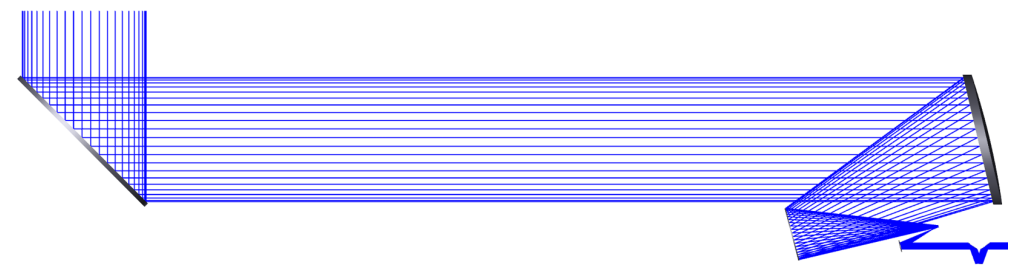

Figure 2. The external optics train (shown here for one arm) which remains unchanged in the new model

\section{SENSITIVITY IMPROVEMENT FROM THEORY}

A conventional FTS works on the principle of interference of light waves. It involves splitting a light beam into two, followed by a delay line mechanism modulating the optical path difference (OPD) between the two beams, which on re-combination and focusing produces interferograms at the image plane. The interferograms are power measurements as a function of OPD. A Fourier transform of the interferograms gives the spectrum of the original beam. FTS is often combined with imaging, such that independent interferograms are obtained simultaneously on each pixel of the detector array together covering a 2-D scene of interest. ${ }^{6,7}$

BETTII also involves an imaging FTS, but instead of splitting a single beam, it uses two beams obtained with two 0.5 meter diameter apertures that are 8 meters apart and pointed at the same astrophysical target. ${ }^{8}$ The vector from one aperture to the other is called a baseline vector. Multiple images are obtained at different baseline vectors with respect to the target source/field. The image intensities as a function of the baseline vectors, and the sky plane intensities are Fourier conjugates in the spatial domain. These multiple images can be used to reconstruct the image of the target at a spatial resolution of $\lambda / 2 B$ where $\lambda$ is the wavelength and $B$ is the baseline length. This is the spatial interferometry aspect of BETTII, and together with the FTS, it is called a double-Fourier interferometer. Thus to summarize, BETTII obtains interferograms for each of the $9 \times 9$ pixels on the detector arrays, and for each of the baselines covered over its duration of observing an astrophysical scene. This data can be used to obtain information about the astrophysical scene at a spatial resolution of $0.5-1^{\prime \prime}$ and a spectral resolution of $\mathrm{R} \sim 50$ in the FIR bands of BETTII.

Rizzo et al.(2015) $)^{9}$ studied the sensitivity of the BETTII instrument, and the effects of intensity noise and phase noise. For $N$ discrete measurements of an interferogram with a step size of $x_{i}$ and a noise variance $\sigma_{i}^{2}$ 
in the interferogram domain, the noise standard deviation in the spectral domain is given by $\sigma_{s}=\sigma_{i} x_{i} \sqrt{N / 2}$. In the interferogram domain, the signal-to-noise ratio can be expressed as $S N R_{i} \equiv I_{0} / \sigma_{i}$, where $I_{0}$ is the peak intensity of the interferogram. For photon noise limited operation, $\sigma_{i} \approx \sqrt{I_{0}}$ and the $S N R_{i}$ expression can be simplified as $S N R_{i}=\sqrt{I_{0}}=\sqrt{\bar{S} w_{s}}$, where $\bar{S}$ is the mean spectral intensity over the spectral bandwidth of interest $w_{s}$. In the spectral domain, we define ${ }^{10}$ the signal-to-noise $S N R_{s} \equiv \bar{S} / \sigma_{s}$, which can be simplified as

$$
S N R_{s}=\frac{\bar{S}}{\sigma_{s}}=\frac{\bar{S}}{\sigma_{i} x_{i} \sqrt{N / 2}}=\frac{1}{x_{i}} \sqrt{\frac{2 \bar{S}}{N w_{s}}}
$$

Using the above relations, we can also derive a relation between the signal-to-noise ratios in the two domains as

$$
S N R_{s}=S N R_{i} \frac{1}{w_{s} x_{i}} \sqrt{\frac{2}{N}}
$$

This shows that even though $S N R_{i}$ is directly proportional to $\sqrt{w_{s}}, S N R_{s}$ is inversely proportional to $\sqrt{w_{s}}$. In other words, on decreasing the spectral bandwidth, even though the central fringe has lesser SNR, the fringe envelope is more extended resulting in more number of fringes being detected over the noise. This is the concept behind using a dispersive element in the BETTII optics train to improve the spectral SNR. The concept has been studied previously by multiple authors. ${ }^{11-13}$ A mathematical treatment including the effect of readout noise is given in Pritt et al. (1997). ${ }^{10}$

\section{INTERFEROMETRIC SIMULATIONS}

We modeled the outputs of BETTII to understand the instrument response to various input fields and to study the effects of various errors, such as intensity noise, OPD noise and filtering. ${ }^{14}$ Using the propagation of electromagnetic fields in the two arms, we simulated the SW and LW detector outputs in the form of interferograms for each pixel, and for each baseline. The simulation also serves as a test-bed to test effects of design modifications, and for devising a technique to derive astrometric and spectroscopic information iteratively from the raw data.

The dispersive grating concept was implemented in this simulation to compare the effects with that of the previous design and with the theoretical results. Figures 3 and 4 show the results for a single point source with a model spectra. The spectra includes a representative transmission function for the BETTII bands. The BETTII plate scale ensures Nyquist sampling of the point spread function (PSF). We carried out the simulations for the source to be at the center of the field of view (FOV) and used only the central pixel interferogram (having most of the signal) in the non-dispersive case for the spectral analysis. The dispersive grating spreads the signal along the columns, according to wavelength. In this case, we used all the interferograms in the central column to recover the spectra. In both cases, the photon noise was approximated as a normal distribution with a standard deviation of $\sqrt{I}$, where $I$ is the signal intensity at the pixel and the OPD.

As is evident from the figures, for the dispersed case, the interferograms include more detectable fringes and consequently the spectra has lesser noise compared to the non-dispersed case. The absorption features in the spectra are identifiable in the new design, but not in the previous design. Since grating dispersion is proportional to wavelength and not wavenumber, there is a slight deviation from the theoretical SNR improvement. The higher wavelengths have greater improvement in spectral SNR than the lower wavelengths within a band. Overall, the spectral SNR improves by a factor of 2.8 when dispersing the signal over 8 pixels in the BETTII TES detectors and considering photon noise as the only noise source. However in reality, the lower the recorded intensities, the more important thermal background noise and detector noise becomes relative to the interferogram signal. Also other errors like phase errors and filtering effects which independently contribute to the overall spectral noise, result in lowering the effective spectral SNR.

The analysis from the interferograms shown here does not represent the actual spectral extraction process, which would require iterative modeling. However, this technique combined with preliminary astrometry can produce the starting model for the iterations. The use of dispersion makes the disentanglement of multiple sources more challenging. In a more complex field, the interferograms of two sources may overlap, not only based on their projected distances along the baseline vector, and/or because of partially overlapping PSFs, but also based on the spectral energy distributions of the individual sources. Information from multiple baselines obtained through field rotation can be used to break these degeneracies. 

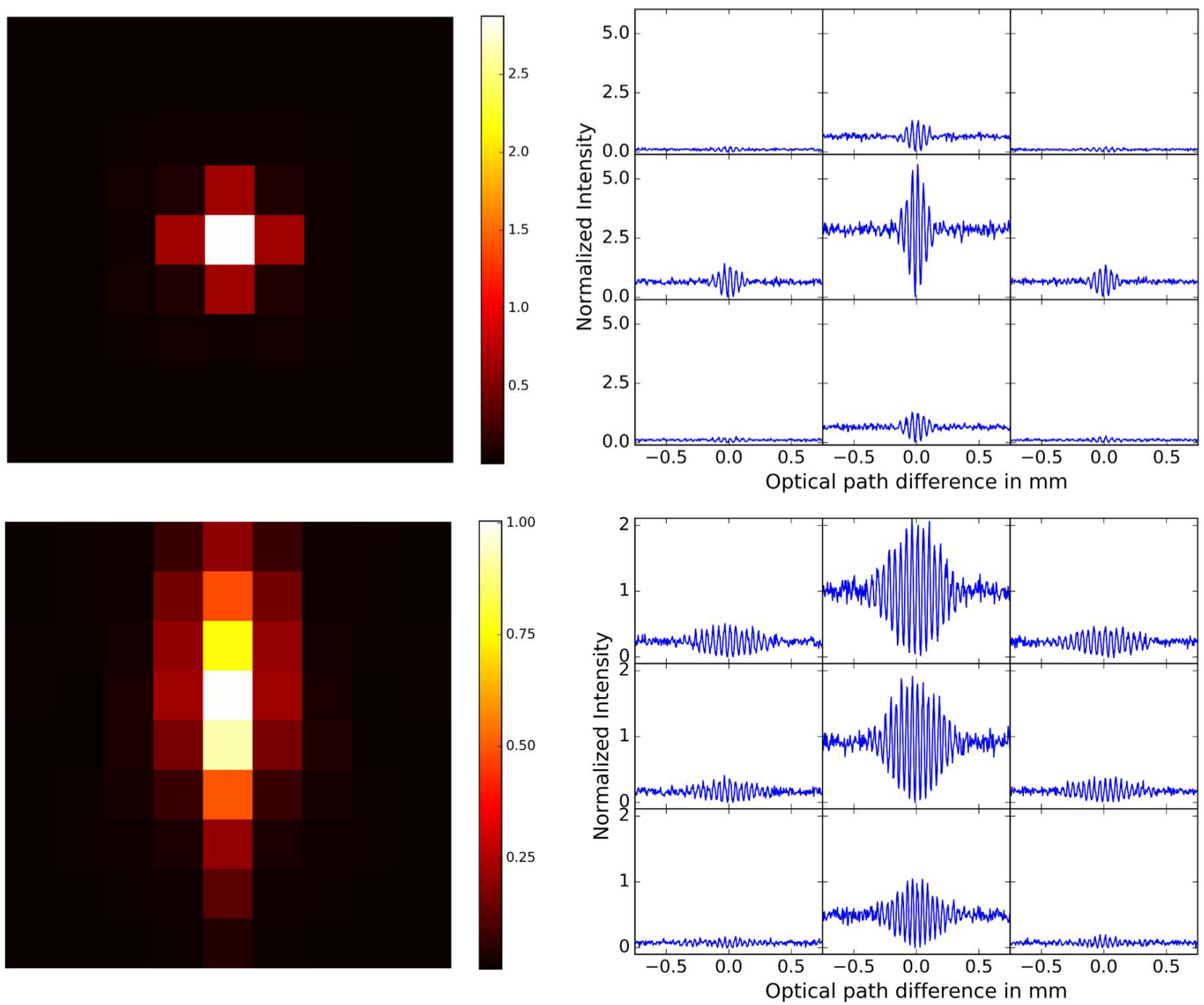

Figure 3. BETTII interferometry simulations for a point source at the center of the field of view (FOV) with an assumed spectra in the SW band. The upper row shows the results for the original BETTII design with no dispersive element, while the lower row represents the results for the new design with a dispersive back-end. The images on the left show the time-averaged and normalized intensity at each pixel of the $9 \times 9 \mathrm{SW}$ detector array. The images on the right show the interferograms for the $3 \times 3$ central pixels of the detector. In the case of the dispersed interferograms, the average intensity of each pixel is lower, but the fringe packets are much wider, resulting in an overall increase in the SNR. 

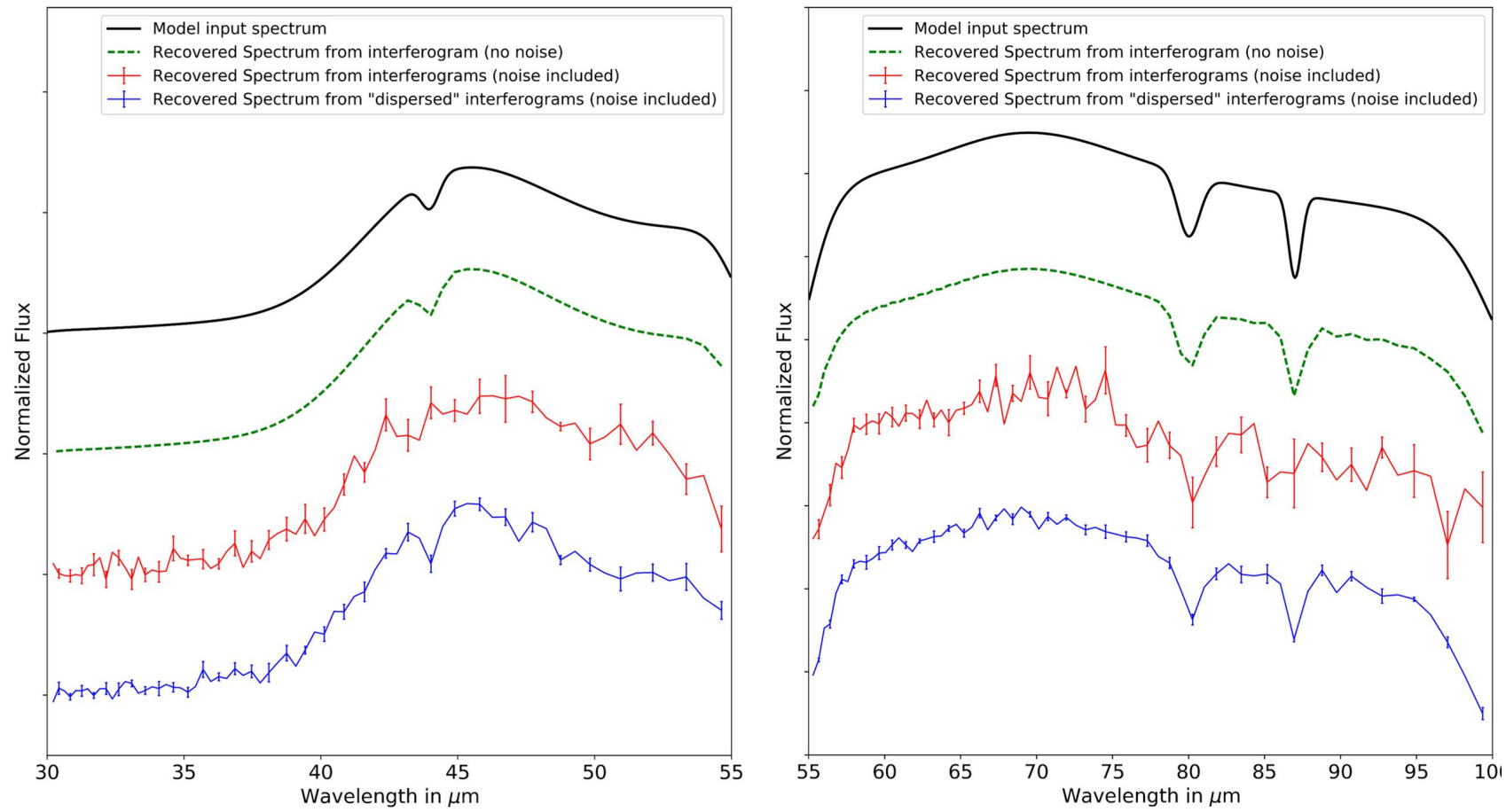

Figure 4. Model input spectra and recovered spectra for SW band (left) and LW band (right). The source is assumed to be in the center of the BETTII FOV. The recovered spectra are shown for (i) interferograms with no noise, (ii), interferograms with photon noise in the no dispersion case and (iii) interferogram with photon noise in the dispersed case. In the non-dispersive case, only the central pixel interferogram is used to extract the spectral information. In the dispersive case, the interferograms for the central column of pixels (see figure 3 lower-left panel) are used for obtaining spectral information. The extracted spectra in the dispersive case have better SNR than the non-dispersive case.

\section{DISPERSIVE BACKEND DESIGN}

The optics was re-modeled in Zemax (as shown in figure 5). The dispersive element is required to be placed after the dichroic separating the two BETTII bands at $55 \mu \mathrm{m}$. In the previous model, the dichroic separation was occurring in non-collimated space. Modeling showed that placing a reflective grating between the dichroic and the detector plane for all 4 outputs is able to disperse the sources along the columns of the detector arrays (see figure 6). A grating of 2.79 lines $/ \mathrm{mm}$ for the $\mathrm{SW}$ band linearly disperses the wavelengths $30 \mu \mathrm{m}$ to 55 $\mu \mathrm{m}$ over $8 \mathrm{~mm}$ on the square detector array of sides $9 \mathrm{~mm}$. The first order diffraction requires a blaze angle of $4.60^{\circ}$ to maximize the efficiency of the waveband, producing a minimum relative efficiency of $78 \%$ at the band extremes. For the LW band, the grating spacing is 2.58 lines $/ \mathrm{mm}$, the blaze angle is $5.84^{\circ}$ and the minimum relative efficiency is $73 \%$. The non-linear effects of not being in the collimated space are negligible for the small range of wavelengths involved here. The SW and LW band specifications are summarized in Table 1.

Some diagnostics for the two channels such as the footprint, spot diagram and wavefront distortions are shown in figures 7, 8 and 9 respectively. The SW band has greater aberrations even after introduction of a toroidal mirror to control astigmatism. However they don't affect the interferograms appreciably. Although the spot is distorted, it is still well within the diffraction limited airy disk over the entire FOV.

The optics train has a focus inside the cryostat prior to the cold pupil. A slit needs to be introduced here to obtain the spectra of sources, that are point-like based on the aperture diffraction limit, but has extended structure in the interferometer resolution limit -for example, individual protostars having a disk and an envelope around them, whose physical parameters we want to study. If a slit is not used, we will retain the full FOV, but full spectral information would be obtained only for sources in a narrow central part of the FOV. More importantly, the background noise contribution will be much higher, thereby nullifying the spectral sensitivity 


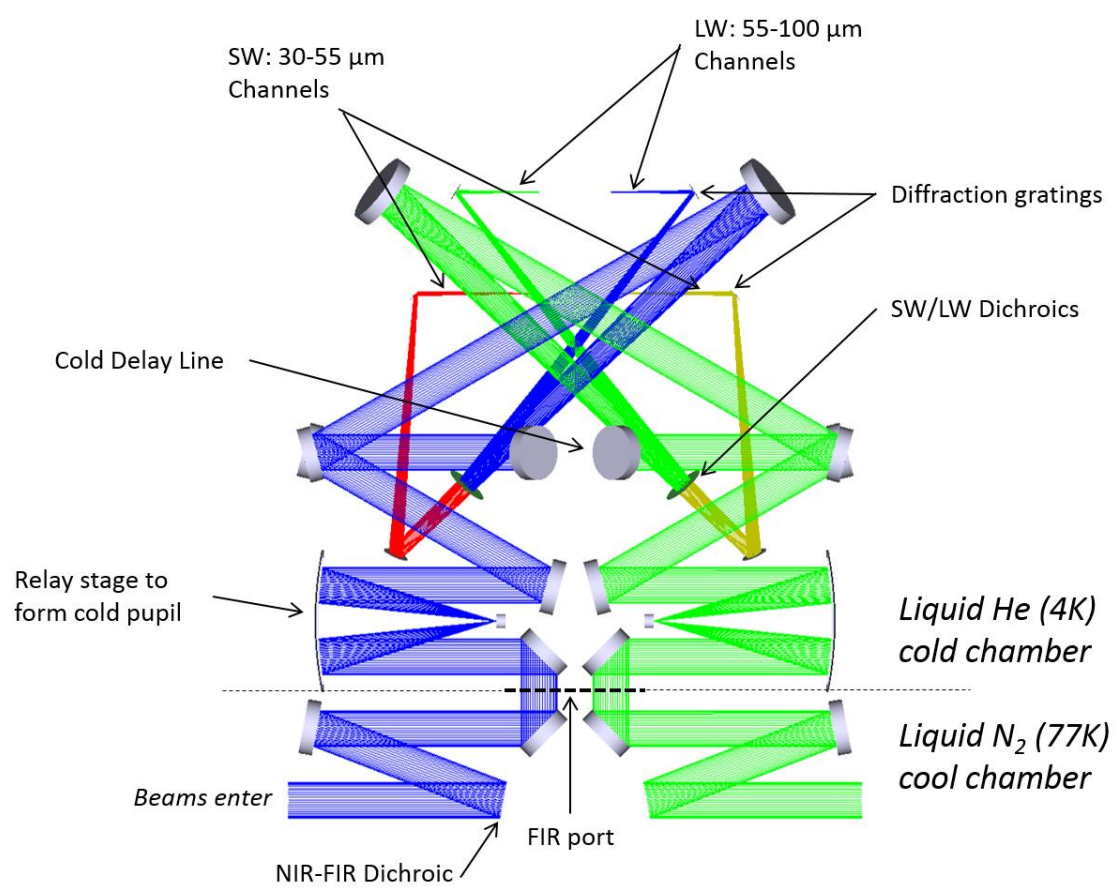

Figure 5. New internal optics model showing salient features

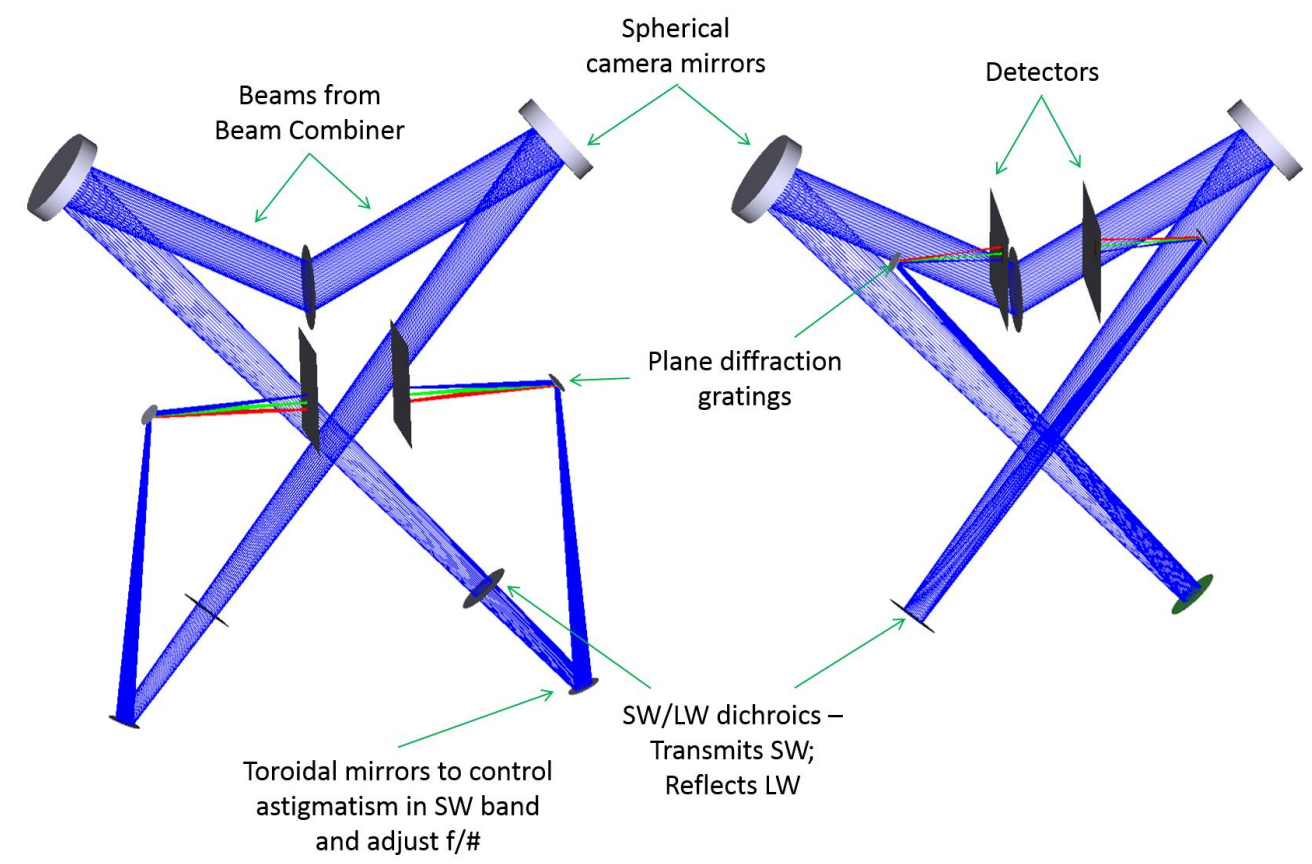

Figure 6. Post Beam-combiner optics for the two BETTII channels :SW (left) and LW(right). 

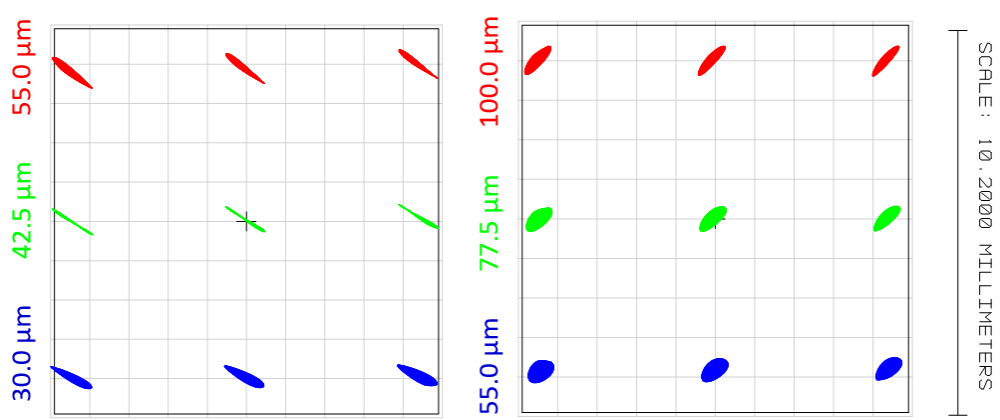

Figure 7. Footprint Diagram at the SW (left) and LW (right) detector planes for three wavelengths and three pointings across the field of view.

OBJ : $-0.0167,0.0000(D E G)$

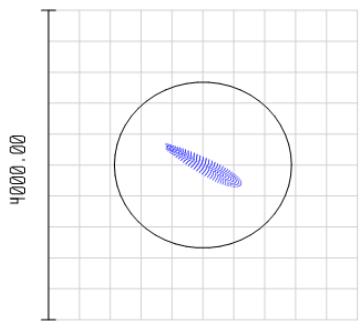

IMA: $-4.521,-4.071 \mathrm{MM}$

OBJ : $-0.0208,0.0000$ (DEG)

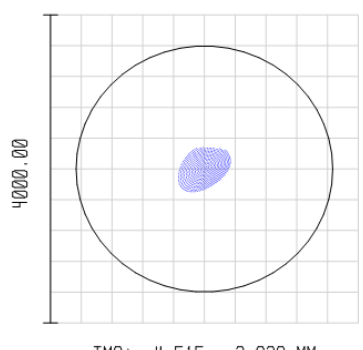

IMA: $-4.515,-3.939 \mathrm{MM}$
OBJ : $0.0000,0.0000(D E G)$

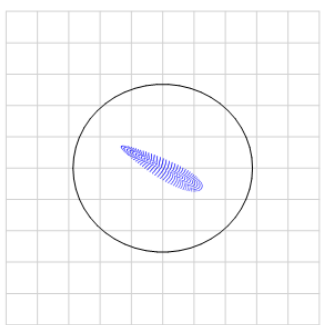

IMA: $-0.045,-4.032 \mathrm{MM}$

OBJ : $0.0000,0.0000$ (DEG)

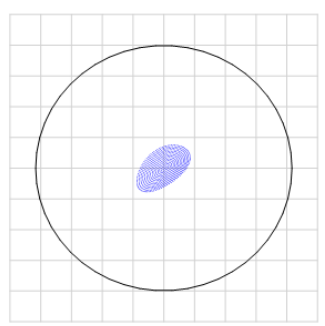

IMA: $-0.009-3.901 \mathrm{MM}$
OBJ : $0.0167,0.0000(D E G)$

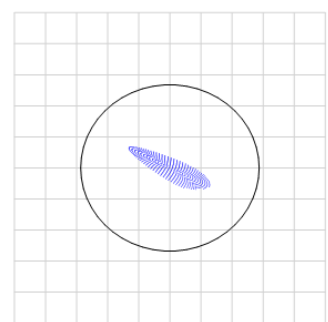

IMA: $4.454,-3.996$ MM

OBJ : $0.0208,0.0000$ (DEG)

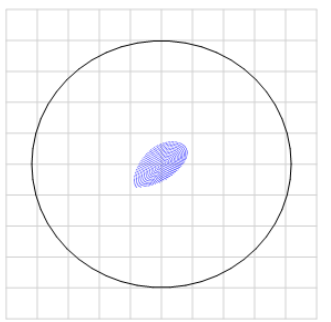

IMA: $4.502,-3.863 \mathrm{MM}$

Figure 8. Spot diagrams at the detector plane for $30 \mu \mathrm{m}$ (top row) and $55 \mu \mathrm{m}$ (bottom row) corresponding to the lowest wavelengths of the two channels. The three columns correspond to pointings at the two edges and the center of the field of view. The circles represent the airy disks at the respective wavelengths with radii $1.11 \mathrm{~mm}$ for $30 \mu \mathrm{m}$ and $1.63 \mathrm{~mm}$ for $55 \mu \mathrm{m}$. 

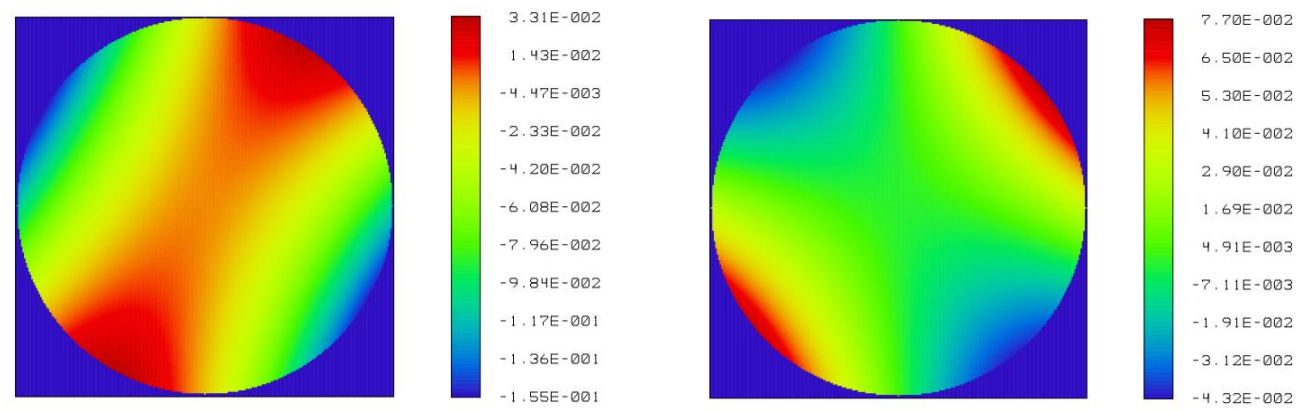

Figure 9. Wavefront functions for $30 \mu \mathrm{m}$ (left) and $55 \mu \mathrm{m}$ (right) having rms values of 0.0420 waves and 0.0246 waves respectively.

Table 1. SW and LW band specifications

\begin{tabular}{|l|l|l|}
\hline & SW band: $30-55 \mu \mathrm{m}$ & LW band: $55-100 \mu \mathrm{m}$ \\
\hline Plate scale & $13.3^{\prime \prime} /$ pixel & $16.7^{\prime \prime} /$ pixel \\
\hline Focal length & $15.45 \mathrm{~m}$ & $12.40 \mathrm{~m}$ \\
\hline f/number & $\mathrm{f} / 30.9$ & $\mathrm{f} / 24.8$ \\
\hline Field of View & $2^{\prime} \times 2^{\prime}$ & $2.5^{\prime} \times 2.5^{\prime}$ \\
\hline Linear Dispersion & $3.125 \mu \mathrm{m} / \mathrm{mm}$ & $5.625 \mu \mathrm{m} / \mathrm{mm}$ \\
\hline
\end{tabular}

gain from dispersing. Additionally, in the no-slit case, the dispersion will result in overlap of fringes from multiple sources corresponding to different parts of the spectra. The disentangling of the spatio-spectral information is more complex in this scenario.

\section{OTHER OPTICS DESIGN CHANGES}

In addition to the dispersive backend, a number of changes are incorporated in the new optics design. In the previous design, each of the two input beams first entered a $4 \mathrm{~K}$ liquid He cooled chamber of the cryostat, where a dichroic divided it into a NIR part (for the tracking channel) and a FIR part (for the science channels). The FIR part continued in the He chamber until the detectors, while the NIR was relayed to a $77 \mathrm{~K}$ liquid $\mathrm{N}_{2}$ cooled chamber. This resulted in a heavy thermal loading on the He cooled chamber. The current model houses the dichroic in the liquid $\mathrm{N}_{2}$ cooled chamber, such that the compressed beam from the external optics enters the 77 $\mathrm{K}$ chamber of the cryostat first (see figure 5). The thermal blockers present here will absorb the unwanted IR radiation, and will deposit lesser heat in the more critical $4 \mathrm{~K}$ chamber housing the detectors. This will lead to longer cryogen hold time and improved detector performance.

Another important modification also involves the entry of light onto the FIR optics bench. Obtaining interferometric fringes in the laboratory using coherent and collimated FIR light is valuable to test the performance of the instrument prior to launch. In the previous model, the two input beams were at diametrically opposite points of the cryostat. In such a case, feeding a coherent FIR beam into both the arms was extremely challenging, because of the requirement of a beam splitting and relaying assembly. The entire input assembly required to be in a cold chamber (to eliminate thermal IR radiation). Further, this assembly would have introduced additional sources of error that would have required disentangling from the instrument errors to determine the instrument performance. The current design bypasses these problems by having two parallel and adjacent input beams into the liquid $\mathrm{He}$ cooled chamber. Thus, for laboratory testing, a collimated FIR beam of diameter roughly 3 times that of the input beam can be used to generate coherent inputs for both arms simultaneously through the FIR port (see figure 5). 
Some of the optics have been re-designed to ensure that there is only one reflective surface per physical optical element. Mirrors like the double-sided focusing parabola were challenging to align for both arms simultaneously because of the presence of non-negligible tip-tilt errors of one reflective surface with respect to the other. The new design avoids such mirrors. The detector planes have been re-oriented to ensure that the detector packaging containing all the four detector arrays has a regular cuboidal shape. The modifications have been checked for compatibility with the mechanical model, and are being implemented on BETTII-2.

\section{REFERENCES}

[1] Rinehart, S. A., Rizzo, M., Benford, D. J., Fixsen, D. J., Veach, T. J., Dhabal, A., Leisawitz, D. T., Mundy, L. G., Silverberg, R. F., Barry, R. K., Staguhn, J. G., Barclay, R., Mentzell, J. E., Griffin, M., Ade, P. A. R., Pascale, E., Klemencic, G., Savini, G., and Juanola-Parramon, R., "The Balloon Experimental Twin Telescope for Infrared Interferometry (BETTII): An Experiment for High Angular Resolution in the Far-Infrared," Publications of the ASP 126, 660-673 (July 2014).

[2] Dhabal, A., Rinehart, S. A., Rizzo, M. J., and Mundy, L., "Optics of Balloon Experimental Twin Telescope for Infrared Interferometry (BETTII): delay lines and alignment," in [Proceedings of the SPIE, Volume 9907, id. $99070 T$ (2016)], 9907 (July 2016).

[3] Dhabal, A., Rinehart, S. A., Rizzo, M. J., Mundy, L., Sampler, H., Juanola-Parramon, R., Veach, T., Fixsen, D., de Lorenzo, J. V. H., and Silverberg, R. F., "Optics alignment of a balloon-borne far-infrared interferometer BETTII," Journal of Astronomical Telescopes, Instruments, and Systems 3 (Apr. 2017).

[4] Rinehart, S. A., Dhabal, A., Fixsen, D. J., Juanola-Parramon, R., Leisawitz, D. T., Maher, S. F., Mentzell, J. E., Mundy, L. G., Rizzo, M. J., Sampler, H., Sharp, E. H., Silverberg, R., Torres, M. C., Veach, T. J., Vila Hernandez de Lorenzo, J., Ade, P., Tucker, C., Pascale, E., and Giorgio, S., "The Balloon Experimental Twin telescope for infrared interferometry (BETTII): first flight," in [Ground-based and Airborne Telescopes VII], Proceedings of the SPIE 10700 (June 2018).

[5] Vila Hernandez de Lorenzo, J., Rinehart, S. A., Nehmetallah, G., Maher, S. F., Rizzo, M. J., Dhabal, A., Fixsen, D. J., Torres, M. C., Veach, T. J., and Esteves, F. M., "Flight performance of the attitude control system of the balloon experimental twin telescope for infrared interferometry (BETTII)," in [Ground-based and Airborne Telescopes VII], Proceedings of the SPIE 10700 (June 2018).

[6] Bennett, C. L., Carter, M. R., Fields, D. J., and Hernandez, J. A. M., "Imaging Fourier transform spectrometer," in [Proceedings of the SPIE, Volume 1937, p. 191-200 (1993).], 1937, 191-200 (Sept. 1993).

[7] Schumann, L. W. and Lomheim, T. S., "Infrared hyperspectral imaging Fourier transform and dispersive spectrometers: comparison of signal-to-noise-based performance," in [Proceedings of the SPIE, Volume 4480, p. 1-14 (2002).], 4480, 1-14 (Jan. 2002).

[8] Mariotti, J.-M. and Ridgway, S. T., "Double Fourier spatio-spectral interferometry - Combining high spectral and high spatial resolution in the near infrared," Astronomy and Astrophysics 195, 350-363 (Apr. 1988).

[9] Rizzo, M. J., Mundy, L. G., Dhabal, A., Fixsen, D. J., Rinehart, S. A., Benford, D. J., Leisawitz, D., Silverberg, R., Veach, T., and Juanola-Parramon, R., "Far-Infrared Double-Fourier Interferometers and their Spectral Sensitivity," Publications of the ASP 127, 1045-1060 (Oct. 2015).

[10] Pritt, Alfred T., J., Kupferman, P. N., Young, S. J., and Keller, R. A., "Imaging LWIR spectrometers for remote sensing applications," in [Proceedings of the SPIE, Volume 3063, p. 138-149 (1997).], 3063, 138-149 (June 1997).

[11] Graham, J. R., "An Imaging Fourier Transform Spectrometer for NGST," ArXiv e-prints (July 1999).

[12] Nordgren, T. E. and Hajian, A. R., "A New Multichannel Fourier Transform Spectrometer," in [Precise Stellar Radial Velocities, ASP Conference Series 185, IAU Colloquium 170. Eds. J. B. Hearnshaw and C. D. Scarfe. ISBN: 1-58381-011-0 (1999), p. 36], 185, 36 (Jan. 1999).

[13] Miller, P. J. and Harvey, A. R., "Signal-to-noise analysis of various imaging systems," in [Proceedings of the SPIE, Volume 4259, p. 16-21 (2001).], 4259, 16-21 (July 2001).

[14] Dhabal, A., Mundy, L. G., Rizzo, M., Rinehart, S., and Juanola-Parramon, R., "Simulations and Interpretations of BETTII Observations," in [American Astronomical Society, AAS Meeting 229, id.155.14], (Jan. 2017). 